\title{
The Feasibility Analysis of Cucumber Disease Prediction with Virtual Reality Technique
}

\author{
Tianchi Zhao ${ }^{1}$, Peng Yuan ${ }^{2, a}$ \\ ${ }^{1}$ NO.2 Middle School of Shijiazhuang, Shijiazhuang 050000, China; \\ ${ }^{2}$ School of Control Science and Engineering, Hebei University of Technology, Tianjin 300401,China. \\ ahebutyuanpeng@126.com
}

Keywords: Image segmentation, characteristic value extraction, significant difference, image space, virtual reality technology

\begin{abstract}
In order to find out the high correlative factors of the crop disease from the images, and to predict the disease process, the author analyze the digital images of cucumber leaf which collected in the process of normal and disease by computer image processing technology. By comparing the nine parameters of the three kinds of color spaces which were in common use, it was found that three of them have significant differences. In the further statistic study, the optimal distinguish intervals of the three parameters were found. All of the distinguish rates of $\mathrm{G}$ (green), V (brightness) and $\mathrm{Cr}$ (red with the difference between the reference value) were more than $80 \%$, and the optimal distinguish interval of them was 149bit, 152bit, 110bit, which can be used as the feature parameters in predicting the process of cucumber crop disease, and become an important reference in the establishment of the prediction model. The test results show that it is feasible to combined predicting cucumber crop disease process by the color analysis of the digital images with showing the process dynamically in display devices by virtual reality technology, which enables the viewer to observe the overall disease process of the crops, and to have a more intuitive feeling of the pathological changes at different time.
\end{abstract}

\section{Introduction}

One of the important applications of computer vision technology in agricultural sector is the crop disease diagnosis by image processing techniques. To find and judge diseases, we can observe the disease symptoms of the leaf. In recent years, with the increasingly perfection and popularity of the digital image processing technology, more and more researchers are using this technology on crop disease diagnosis. Yuan Yuan et al identified the image of target leaf spot and powdery mildew of cucumber, and then proposed the segmentation of cucumber leaf disease spot under complex background $^{[1,2]}$. Zhanwu Peng studied the downy mildew of cucumber by leaf image ${ }^{[3]}$; Xianfeng Wang et al identified the downy mildew, target leaf spot and the anthracnose by the extraction of scab $^{[4]}$. Since the researches mentioned above are focused on the analysis of cucumber which has dominant lesion characteristics, whether can find a method that can predict when the symptoms shows before the appearance of the dominant trait in crops, which is of great significance for reducing the resulting yield and economic losses by prevention and remedy.

Virtual reality (VR) technology, is a new technology to integrate the information of the real world and virtual world. By the science and technology, such as computer, and the simulation and then overlay, the virtual information which is difficult to experience (visual information, sound, smell, touch, etc.) in the real world within a certain time and space, is applied to the real world, so that people can perceive it by the human senses, and then can gain the sensory experience which beyond the reality ${ }^{[5,6]}$. AR technology has brought the revolutionary change in artificial intelligence, CAD and graphic simulation, virtual communication, remote sensing, entertainment, simulation training and many other areas ${ }^{[7,8]}$. Using the feature that the AR technology can overlay the real environment and virtual objects in real-time to the same picture or space, we can show both the real- time scene of the crops and the prediction of the crop disease processes (such as leaf color change process, etc.). On the same screen, we can diagnose the crop disease, on one hand, and show 
the disease pathological changes state vividly, on the other hand. It can make the viewers know more information of specific symptoms of disease in a particular period of time by the use of this technology. For the public, it is an application of entertainment, and for researchers it can be used as an auxiliary reference for disease prediction and research.

In order to achieve the expectation, the author conduct the research on predicting the disease process, extract the color characteristics of the cucumber leaf image at the stages of normal and have dominant lesion appearance. To compare with them, we can verify the feasibility of using color analysis method to predict disease processes, then to establish the lesions prediction model based on computer vision technology, and finally to achieve the accurately and fast predict of the disease of cucumber crop without explicit lesions. Then the feasibility of the combination of the disease process and the virtual reality and display in the device real-timely are discussed.

\section{Materials and Methods}

\subsection{Experiment Time and Location}

The experiment is carried out in the solar greenhouse in hebei academy of agricultural sciences at the time of November - December, 2015. The tested plots are located in north latitude $38^{\circ} 12$ ', longitude $114^{\circ} 38$ ', which belong to the temperate semi-humid continental monsoon climate. The engraftment soil is calcareous -brown soil, which nutrient status are: alkali solution nitrogen is 70 $\mathrm{mg} / \mathrm{kg}$, water-soluble phosphorus is $12 \mathrm{mg} / \mathrm{kg}$, and effective potassium is $115 \mathrm{mg} / \mathrm{kg}$. The field area of the tested plot is $384 \mathrm{~m} 2$, the planting distance is $33 \mathrm{~cm}$, and the line size are $40 \mathrm{~cm}$ and $80 \mathrm{~cm}$. The two ridge cucumber as a group, and each group as a community.

\subsection{Experiment Materials and Equipment}

The variety of the cucumber tested is west phoenix 96-6 grafting, which is homed on October $28^{\text {th }}, 2015$ and pulled up seedlings on March 2ed, 2016.

The experiment equipments include a computer and a network camera. The configurations of the computer are as follow: Intel ${ }^{\circledR}$ Core $^{\mathrm{TM}} \mathrm{i} 5-5200 \mathrm{U}$ processor, $8 \mathrm{~GB}$ of memory installed, and 15 inch LCD monitor. The camera is an automatically convert color CCD camera which model is 6062-13, and the resolution of the image is $1280 * 720$. The camera is installed at the location which $90 \mathrm{~cm}$ from the ground and $130 \mathrm{~cm}$ from the crops.

\subsection{Experiment Methods}

A network camera is used to film the cucumber growth process for a month. From them we select three stages of leaf color digital image such as the leaves in the stage of normal, the stage of early pathological changes of symptoms and the stage of pathological symptoms respectively. The measuring time is November $10^{\text {th }}, 2015$, December $5^{\text {th }}, 2015$ and December $29^{\text {th }}, 2015$.

\section{Color Feature Extraction}

\subsection{Image Preprocessing}

The software of Matlab can save the digital image by frame from video. The VS2010 and OpenCV are utilized to extract the blade part in the images. The RGB color space is one of the most widely used color space, which get all sorts of color by the change of the three color channels(Red, Green, Blue) and the overlapping between them ${ }^{[9,10]}$. The RGB color space is such a kind of color space that related to equipment, which the most common usage is the display system. Since under the RGB color space, the correlations between the components is too big, and each channel include the brightness information, which can easily affected by the surrounding environment (light, etc.), it is not match the process of human cognitive color and not suitable for analysis of color image and segmentation. Compared with RGB space, the HSV color space is from the human visual system, therefore more suitable for image segmentation ${ }^{[11,12]}$. The extraction of the blade in the images in this article is in the HSV space.

In HSV color space, the leaf part which will be studied is extracted. Then transfer them to the RGB color space and summarized the value of 3 channels of RGB at all effective pixels of the leaf part. 
Through this method, we can synchronous implementation the image denoising pretreatment $^{[13,14,15]}$ and the extraction of RGB values, which can greatly simplifies the test steps. The effect of normal leaves is shown in Fig1.

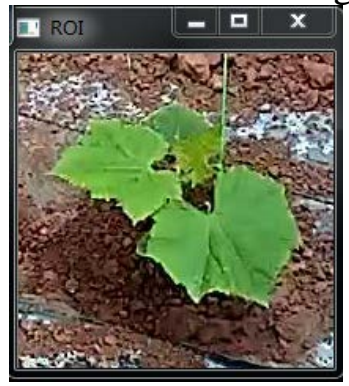

(a) the original leaf in control group

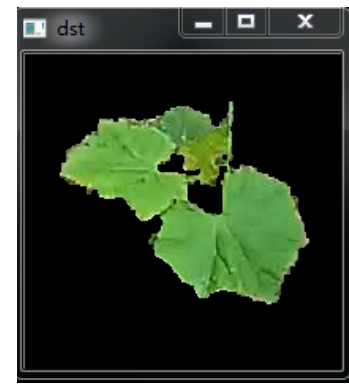

(b) the segmentation leaf in control group

Fig. 1 The effect of leaf blade segmentation

\subsection{The characteristic value Extraction under the RGB Color Space}

The RGB color space distribute an intensity value range from 0-255 for each RGB component by RGB model for each pixel in the image. So that the image color differences can be reflected in the numerical, even the subtle color differences to the naked eye can perform the obvious change in value. Blade will perform tonal changes when they pathogenesis, which normal leaves are green, and the lesion leaves will turn yellow.

We select the normal cucumber leaves in the test plot as control group, and the pathological leaves in the process of two time node as treatment group. From sampling 15 images in each group, we randomly selected 5 images and numbered, in which 1-5 for the control group, 7-11 for the treatment group 1 at first node time, 13-17 for the treatment group 2 at second node time, number 6 , 12, 18 are blank. By color analysis algorithm, the statistical figure for RGB values is shown in Fig2.

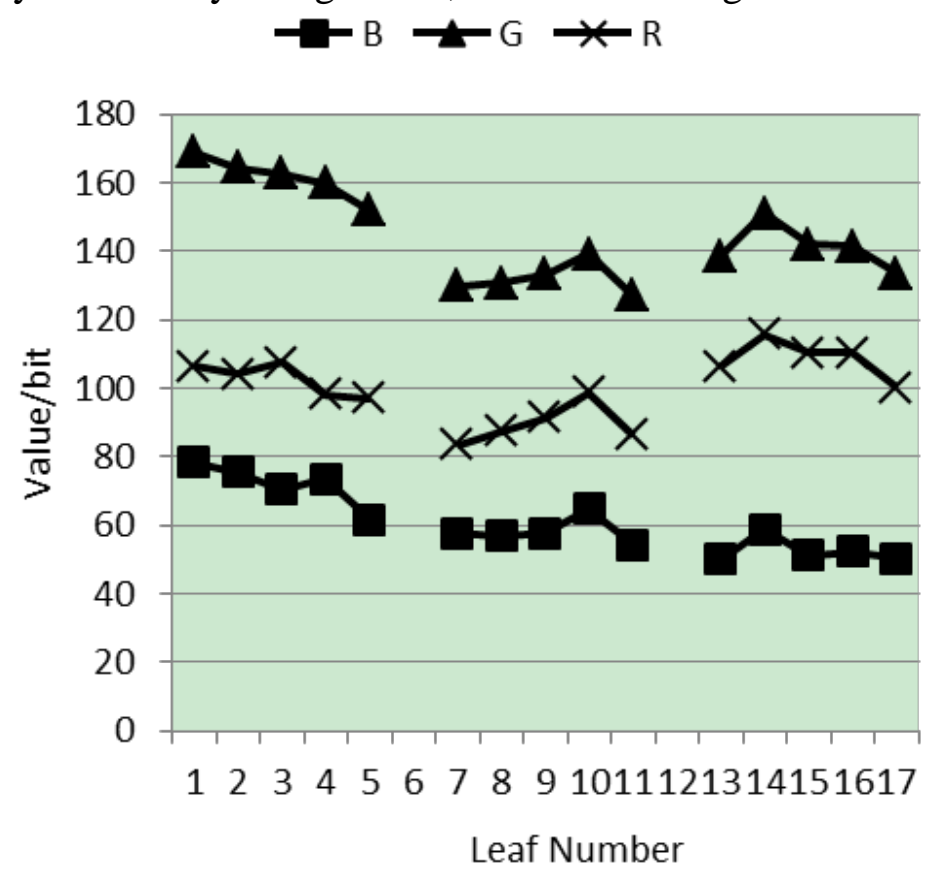

Fig. 2 The statistical figure of R, G, B

In the control group, the range of $\mathrm{R}, \mathrm{G}, \mathrm{B}$ is $95-105,150-170,60-80$ respectively;

In the treatment group 1, the range of R, G, B is 80-100, 130-140, 55-65 respectively;

In the treatment group 2, the range of R, G, B is 100-120, 130-150, 50-60 respectively;

The results of significant difference test of single factor ANOVA and LSD multiple comparison to the data by SPSS of R,G,B value are shown in Table1 and Table2,in which group 1 representative the control group, group 2 representative the treatment group 1,and group 3 representative the treatment group2. 
Table 1 The result of significant difference

\begin{tabular}{ccccc}
\hline & df & Mean & F & Significance \\
\hline B & 2 & 494.600 & 21.238 & 0.000 \\
G & 2 & 1136.698 & 33.316 & 0.000 \\
R & 2 & 484.171 & 15.948 & 0.000 \\
\hline
\end{tabular}

Table 2 The result of LSD multiple comparison

\begin{tabular}{cccc}
\hline Groups & Significant difference of B & Significant difference of G & Significant difference of R \\
\hline $1-2$ & 0.001 & 0.000 & 0.003 \\
$1-3$ & 0.000 & 0.000 & 0.112 \\
$2-1$ & 0.001 & 0.000 & 0.003 \\
$2-3$ & 0.085 & 0.027 & 0.000 \\
$3-1$ & 0.000 & 0.000 & 0.112 \\
$3-2$ & 0.085 & 0.027 & 0.000 \\
\hline
\end{tabular}

Through Table3, in the level of 0.05 , it can be found that the value of $G$ in three groups of comparison shows great significant difference, which can be chosen as characteristic value.

In Fig2,the distinction between $G$ value range is at around 150.By statistic the tonal range on each group of 15 leaf through extract the $\mathrm{G}$ value, it has the largest distinction rate greater than $80 \%$ at 149 in each group(See Table3).

It is concluded that the number of tonal that greater than 149 in control group is $86.67 \%$ and in treatment groups are $0 \%$ and $20 \%$ respectively. The value of $\mathrm{G}$ at 149 can thought to be the characteristic value on whether the leaf lesions or the status of the lesions.

Table 3 Tonal distribution statistics of $\mathrm{G}$

\begin{tabular}{ccccc}
\hline $\mathrm{G}$ & Tonal Range & Num of Tonal $<149$ & Num of Tonal $>=149$ & Distinction rate/\% \\
\hline Control Group & $147-175$ & 2 & 13 & 86.67 \\
Treatment Group 1 & $122-143$ & 15 & 0 & 100.00 \\
Treatment Group 2 & $134-157$ & 12 & 3 & 80.00 \\
\hline
\end{tabular}

\subsection{The characteristic value Extraction under the HSV Color Space}

HSV color space is from the human visual system, which describe color in color tone (Hue), saturation(Saturation, Chroma) and brightness(Intensity or Brightness) ${ }^{[1,2,3,4]}$.The hue and saturation are known as chroma, which are usually used to represent the class of color and the degree of depth. As the sensitivity of brightness in human visual is far stronger than the sensitive degree of color shade, human visual system often use HSV color space to facilitate the color processing and recognition. It is more suitable for human visual properties than RGB color space. In image processing and computer vision a large number of algorithms can be used conveniently in HSV color space, which can be handled separately and is independent of each other. In HSV color space, we can greatly simplify the workload of image analysis and processing.

Then count the values of HSV converted from RGB values.

The results of significant difference test of single factor ANOVA and LSD multiple comparison to the data from last step by SPSS are shown in Table4 and Table5.

Table 4 The result of significant difference

\begin{tabular}{ccccc}
\hline & df & Mean & F & Significance \\
\hline H & 2 & 308526.085 & 35.274 & 0.000 \\
S & 2 & 0.008 & 25.944 & 0.000 \\
V & 2 & 1136.698 & 33.316 & 0.000 \\
\hline
\end{tabular}

Table 5 The result of LSD multiple comparison

\begin{tabular}{cccc}
\hline Groups & Significant difference of H & Significant difference of S & Significant difference of V \\
\hline $1-2$ & 0.881 & 0.764 & 0.000 \\
$1-3$ & 0.000 & 0.000 & 0.000 \\
$2-1$ & 0.881 & 0.764 & 0.000 \\
$2-3$ & 0.000 & 0.000 & 0.027 \\
$3-1$ & 0.000 & 0.000 & 0.000 \\
$3-2$ & 0.000 & 0.000 & 0.027 \\
\hline
\end{tabular}


Through Table5, in the level of 0.05 , it can be found that the value of $\mathrm{V}$ in three groups of comparison shows great significant difference, which can be chosen as characteristic value.

By the same analysis on each group of 15 leaf through extract the $\mathrm{V}$ value and statistic the tonal range, it has the largest distinction rate at 152 in each group.

The result of analyzing the distribution of tonal of $\mathrm{V}$ shows in Table6,in which the number of tonal that greater than 152 in control group is $80 \%$ and in treatment groups are $0 \%$ and $6.77 \%$ respectively.

Table 6 Tonal distribution statistics of $\mathrm{V}$

\begin{tabular}{ccccc}
\hline $\mathrm{V}$ & Tonal Range & Num of Tonal $<152$ & Num of Tonal $>=152$ & Distinction rate /\% \\
\hline Control Group & $147-175$ & 3 & 12 & 80.00 \\
Treatment Group 1 & $122-143$ & 15 & 0 & 100.00 \\
Treatment Group 2 & $134-157$ & 14 & 1 & 93.33 \\
\hline
\end{tabular}

\subsection{The characteristic value Extraction under the YCbCr Color Space}

$\mathrm{YCbCr}$ color space is widely used in digital video image, which is the common space in the JPEG images. Y in the system to store intensity information, and $\mathrm{Cb}, \mathrm{Cr}$ store the color information of the image. $\mathrm{Cb}$ is for the difference between blue and reference, and $\mathrm{Cr}$ is for the difference between red and reference value.

Then count the values of YCbCr space converted from RGB space values.

The results of significant difference test of single factor ANOVA and LSD multiple comparison to the data from last step by SPSS are shown in Table7 and Table8.

Table 7 The result of significant difference

\begin{tabular}{ccccc}
\hline & df & Mean & F & Significance \\
\hline $\mathrm{Y}$ & 2 & 637.517 & 22.033 & 0.000 \\
$\mathrm{Cb}$ & 2 & 501.465 & 19.876 & 0.000 \\
$\mathrm{Cr}$ & 2 & 211.152 & 105.326 & 0.000 \\
\hline
\end{tabular}

Table 8 The result of LSD multiple comparison

\begin{tabular}{cccc}
\hline Groups & Significant difference of Y & Significant difference of Cb & Significant difference of Cr \\
\hline $1-2$ & 0.000 & 0.000 & 0.000 \\
$1-3$ & 0.004 & 0.000 & 0.000 \\
$2-1$ & 0.000 & 0.000 & 0.000 \\
$2-3$ & 0.010 & 0.296 & 0.000 \\
$3-1$ & 0.004 & 0.000 & 0.000 \\
$3-2$ & 0.010 & 0.296 & 0.000 \\
\hline
\end{tabular}

Through Table8, in the level of 0.05 , it can be found that the value of $\mathrm{Cr}$ in three groups of comparison shows great significant difference, which can be chosen as characteristic value.

The result of analyzing the distribution of tonal of Cr shows in Table9,in which the number of tonal that greater than 110 in control group is $100 \%$ and in treatment groups are $99.33 \%$ and $100 \%$ respectively.

Table 9 Tonal distribution statistics of $\mathrm{Cr}$

\begin{tabular}{ccccc}
\hline Cr & Tonal Range & Num of Tonal $<110$ & Num of Tonal $>=110$ & Distinction rate $/ \%$ \\
\hline Control Group & $71-82$ & 15 & 0 & 100.00 \\
Treatment Group 1 & $84-91$ & 1 & 14 & 93.33 \\
Treatment Group 2 & $92-100$ & 0 & 15 & 100.00 \\
\hline
\end{tabular}

\section{Conclusion}

By analysis and comparison of nine kinds of color parameters in three kinds of commonly used color space, the experiment found significant differences between the leaf blade of normal and in the process of the lesions in three parameters of G, V, Cr. It is also found that, by the statistic study on the tonal, the optimal point of $\mathrm{G}$ value is 149 , of $\mathrm{V}$ is 152 , of $\mathrm{Cr}$ is 110 , at which the distinction rate between the blades is higher than $80 \%$.So that, the three parameters can be used in cucumber 
crop disease state prediction model as identification parameters.

\section{Discussion}

In recent years, as the rapid development of image processing, pattern recognition technology, it is very possible to use the computer vision technology in forecast and diagnosis of crop disease which will become a hot research direction. It can be seen from the author's research, the use of color analysis on crop which did not appear dominant characteristics of pathological changes is feasible to predict crop disease processes, this conclusion opens up a new method which can use effective remedial measures to save the crops before irreparable loss, to reduce costs, and to improve the efficiency of planting It break the limitation of only having lesion characteristics of crops for later research. At the same time, this research makes great significance in practical research and lay the foundation for the establishment of quick disease diagnosis system when the crops did not have explicit pathological expressions.

In addition, in the current worldwide, as a new man-machine interface and simulation tools, AR earns a lot of increasingly widespread attentions, and has played an important role, shown great potential. It becomes to a powerful way for human intelligent extension, and makes a far-reaching influence of production and social life. In China, virtual reality is still in its infancy. Many enterprises which fields are virtual reality have begun to focus on the development and application of "virtual reality". According to the research conclusion of this article, it is feasible to predict the crop disease processes and then to display it with the virtual reality. The combination of prediction and virtual reality is an audacious attempt, not only to provide a new auxiliary way for scientific research, but also to propose a new application in social life, which increase the entertaining and the science and education for the current virtual product.

\section{References}

[1] Yuan Yuan, Miao Li, Sheng Chen, et al. Segmentation of Cucumber Leaf Disease Image with Complex Background[J]. Transactions of the Chinese Society for Agricultural Machinery, Vol. 44 (2008) No. 10, p. 233-237.

[2] Yuan Yuan, Miao Li, Na Wu, et al. Research on Evaluation Methods for Image Segmentation of Cucumber Disease [J]. Agricultural mechanization research, Vol. 10 (2014), p. 160-163.

[3] Zhanwu Peng, Xiuli Si, Xue Wang, et al. Extraction of Cucumber Diseases Based on Computer Image Processing Technology[J]. Agricultural mechanization research, Vol. 2 (2014), p. 179-187.

[4] Xianfeng Wang, Shanwen Zhang, Zhen Wang, et al. Recognition of cucumber diseases based on leaf image and environmental information[J]. Transactions of the Chinese Society of Agricultural Engineering, Vol. 30 (2014) No. 14, p. 148-153.

[5]Zhou Zhong, Zhou Yi, Xiao JiangJian. The Review of Virtual Reality Technique[J]. Chinese Science: Information Science, Vol. 45 (2015) No. 2, p. 157-180.

[6]Wu Fengjuan. The Application of Virtual Reality Technology[J]. Science \& Technology Vision, Vol. 7 (2015) p. 104-104.

[7] Kong Liwen, Xue Zhaojun, Chen Long. Brain-machine Interface Based on Virtual Reality Technology Research Progress[J]. Journal of Electronic Measurement and Instrument, Vol. 3(2015), p. 317-327.

[8]Chen Haolei, Zou Xiangjun, Chen Yan, et al. The Latest Development of Virtual Reality Technology and Prospect[J]. Science paper Online, Vol. 1(2011), p. 1-5+14.

[9] Garcia-Mateos, J.L.Hernandez-Hernandez, D.Escarabajal-Henarejos, et al. Study and comparison of color models for automatic image analysis in irrigation management applications[J]. 
Agricultural Water Management, Vol. 151(2015), p. 158-166.

[10] Jun Li, Zhende Chen. Study on the Color Analysis of Tomato Yellow Leaf Curl Virus Disease Based on Digital Images[J]. Chinese Agricultural Science Bulletin, Vol. 29(2013) N0. 31, p. 96-100.

[11] Yinghua Guo. Image Segmentation Based on HSV Color Space[J]. Heilongjiang Metallurgy, Vol. 31(2011) No.2, p. 35-37.

[12] Jianjun Yin, Xinzhong Wang, Hanping Mao, et al. Contrasitive Research on Tomato Image Segmentation Under RGB and HIS Color Space[J]. Agricultural mechanization research, Vol. 11(2006), p. 171-174.

[13] Qingchun Feng, Wei Cheng, Qinghua Yang, et al. Identification and localization of overlapping tomatoes based on linear structured light vision system[J]. Journal of China Agricultural University, Vol. 20(2015) No.4, p. 100-106.

[14] Peng Guo, Naixiang Li. Discussing on Development of Agriculture Modernization Supporting by Modern Intelligent Agriculture Technologies[J]. Agricultural mechanization research, Vol. 8(2014), p. 10-18.

[15] Teng Hang, Hanping Mao, Xiaodong Zhang,J et al. Nondestructive Testing of Tomato Growth Information Based on Machine Vision[J]. Agricultural mechanization research, Vol. 11(2015), p. 192-197. 